\title{
Presupposition and the a priori
}

\author{
Nate Charlow
}

(C) Springer Science+Business Media B.V. 2012

\begin{abstract}
This paper argues for and explores the implications of the following epistemological principle for knowability a priori (with ' $\mathcal{K}_{\mathcal{A}}$ ' abbreviating 'it is knowable a priori that').

(AK) For all $\phi, \psi$ such that $\phi$ semantically presupposes $\psi$ : if $\mathcal{K}_{\mathcal{A}} \phi, \mathcal{K}_{\mathcal{A}} \psi$.

Well-known arguments for the contingent a priori and a priori knowledge of logical truth founder when the semantic presuppositions of the putative items of knowledge are made explicit. Likewise, certain kinds of analytic truth turn out to carry semantic presuppositions that make them ineligible as items of a priori knowledge. On a happier note, I argue that (AK) offers an appealing, theory-neutral explanation of the a posteriori character of certain necessary identities, as well as an interesting rationalization for a commonplace linguistic maneuver in philosophical work on the a priori.
\end{abstract}

Keywords A priori - Semantic presupposition - Contingent a priori · Necessary a posteriori $\cdot$ Kripke $\cdot$ Epistemology of logic

\section{Introduction}

In this paper, I argue that the linguistic phenomenon of semantic presupposition is central to epistemological theorizing about the a priori. Specifically, I propose, explain, defend, and apply the following constraint on knowability a priori (with ' $\mathcal{K}_{\mathcal{A}}$ ' abbreviating 'it is knowable a priori that').

(AK) For all $\phi, \psi$ such that $\phi$ semantically presupposes $\psi$ : if $\mathcal{K}_{\mathcal{A}} \phi, \mathcal{K}_{\mathcal{A}} \psi$.

N. Charlow $(\bowtie)$

Department of Philosophy, University of Toronto, 170 St. George Street, Toronto, ON M5R 2M8, Canada

e-mail: nate.charlow@gmail.com 
(AK) claims a sentence's content is knowable a priori only if its semantic presuppositions are too. Section 2 defines the notion of 'semantic presupposition' invoked by (AK). Section 3 makes use of this definition (and some plausible assumptions about the closure of knowability a priori under a priori knowable entailment) to argue in favor of (AK).

The rest of the paper explores the implications of (AK) for the a priori. Wellknown arguments for the contingent a priori and a priori knowledge of logical truth founder when the semantic presuppositions of the putative items of knowledge are made explicit. Likewise, certain kinds of analytic truth turn out to carry semantic presuppositions that make them ineligible to be items of a priori knowledge. On a happier note, I argue that (AK) offers an appealing, theory-neutral explanation of the a posteriori character of certain necessary identities, as well as an interesting rationalization for a commonplace linguistic maneuver in philosophical work on the a priori.

\section{Semantic presupposition}

I'll be using the expression "semantic presupposition" idiosyncratically, so that every semantic presupposition is a "garden-variety" presupposition (i.e., a presupposition in the standard, linguistic sense ${ }^{1}$ ), but not every garden-variety presupposition counts as a semantic presupposition.

Our purposes demand some technical precision about the notion of semantic presupposition that (AK) invokes. First, some familiar definitions and conventions. Let $\alpha$ be a vocabulary item of any syntactic type. Then:

- The character of $\alpha, \llbracket \alpha \rrbracket$, if defined, is a function, possibly partial, from contexts of utterance into intensions.

- The intension of $\alpha$ at a context $c, \llbracket \alpha \rrbracket^{c}$, if defined, is a function, possibly partial, from indices of evaluation (worlds) into extensions.

Let $\phi$ be any well-formed sentence of English (or a suitably regimented formal representation of English). If $\llbracket \phi \rrbracket^{c}$ is defined, then $\llbracket \phi \rrbracket^{c}$ is a function, possibly partial, from worlds to truth-values. If $\llbracket \phi \rrbracket^{c, w}$ is defined, then $\llbracket \phi \rrbracket^{c, w}$ is a truth-value. In sum: ${ }^{2}$

$$
\llbracket \phi \rrbracket=\lambda c: \llbracket \phi \rrbracket^{c} \text { is defined } . \lambda w \cdot\left\{\begin{array}{l}
1 \quad \text { if } \llbracket \phi \rrbracket^{c, w}=1 \\
0 \quad \text { if } \llbracket \phi \rrbracket^{c, w}=0 \\
\text { undefined, otherwise }
\end{array}\right.
$$

Definition 1 clarifies the notion of semantic presupposition invoked by (AK). Let $\alpha$ be any syntactic item, and $w_{c}$ be the world locating $c$. (Contexts, for my purposes, are whatever things fix the values of indexicals like 'actual', 'now', etc. Fixing a

\footnotetext{
${ }^{1}$ For a useful overview of the notion, see von Fintel (2004) and Heim (1991).

2 In the standard $\lambda$-notation, ':' indicates partiality. For example, $\lambda x: F(x) \cdot x$ is a partial identity function, mapping all and only those $x$ 's such that $F(x)$ to themselves.
} 
context means fixing a world - the world locating the context-a time-the time locating the context-etc.)

Definition 1 a semantically presupposes $\psi$ iff either:

(SP1) For all $w$ : if $\llbracket \alpha \rrbracket^{c, w}$ is defined, $\llbracket \psi \rrbracket^{c, w}=1$, or

(SP2) For all $c$ : if $\llbracket \alpha \rrbracket^{c}$ is defined, $\llbracket \psi \rrbracket^{c, w_{c}}=1$

Informally, $\alpha$ semantically presupposes $\psi$ via iff either the intension of $\alpha$ is unevaluable at $\neg \psi$ worlds, or the character of $\alpha$ is unevaluable at $\neg \psi$ contexts. ${ }^{3}$ To illustrate the meaning of clauses (SP1) and (SP2), I will describe some examples.

\subsection{SP1 presupposition: definite descriptions}

It is plausible, if somewhat controversial, that both definite descriptions of the form $\ulcorner$ the $P\urcorner$ and sentences of the form $\ulcorner$ the $P Q\urcorner$ carry (SP1) presuppositions, so that "The $P Q$ expresses a partial proposition which is defined only for worlds [where] there is a unique P" (von Fintel 2004).

Indeed, this follows, more or less directly, from either of the following lexical entries for $\llbracket t h e \rrbracket^{c, w}$ (stated in Heim and Kratzer 1998 and von Fintel and Heim 2007, respectively). Letting $\mathcal{P}=\llbracket P \rrbracket^{c, w}, \mathcal{Q}=\llbracket Q \rrbracket^{c, w}$ :

(1) $\lambda \mathcal{P}: \exists ! x[\mathcal{P}(x)=1] . \lambda \mathcal{Q} \cdot \forall x[\mathcal{P}(x)=1 \rightarrow \mathcal{Q}(x)=1]$

(2) $\lambda \mathcal{P}: \exists ! x[\mathcal{P}(x)=1] . \iota y[\mathcal{P}(y)=1]$

The first lexical entry treats 'the' as a generalized quantifier, while the second treats it as non-quantificational (loosely speaking, referential). But, on either lexical entry for 'the', its extension is a partial function, defined only over those $\mathcal{P}$ such that there is a unique individual that $\mathcal{P}$ maps to 1 . So, for any $w$, $\llbracket$ the $P \rrbracket^{c, w}$ (and, so, $\llbracket$ the $P Q \rrbracket^{c, w}$ ) is undefined unless there is a unique $\mathrm{P}$ in $w .{ }^{4}$

Although controversial, this claim boasts fairly wide acceptance among linguists. I will be assuming its correctness in the remainder of the paper.

\subsection{SP2 presupposition: stipulative definition}

Informally, $\alpha$ semantically presupposes that $\psi$ via clause (SP2) iff the character of $\alpha$ is inevaluable at $\neg \psi$ contexts—iff $\alpha$ cannot be associated with an intension at $\neg \psi$ contexts.

\footnotetext{
3 There may be cases where $\alpha$ and $\psi$ meet condition (SP1) or (SP2) without $\alpha$ presupposing $\psi$. To pick something at random, perhaps it is a condition on a sentence $\phi$ being evaluable for truth that $\llbracket \cdot \rrbracket$ is a compositional function from sentences to characters (in particular, that $\llbracket \phi \rrbracket$ is defined and computable compositionally). We would not generally want to say that $\phi$ presupposed that there is such a compositional interpretation function. Though such cases may be relevant to the possibility of a priori knowledge (indeed, I think they probably are), they are not really my focus here. My point in this paper is to make the case that much philosophical discussion of the a priori has failed to notice the epistemological significance of things that are accurately described as semantic presuppositions.

4 Except in cases of "local presupposition satisfaction." See Sect. 4.3 below.
} 
Cases of stipulative definition apparently involve (SP2) presuppositions. ${ }^{5}$ Suppose we assign the name 'Julius' a referent by stipulating (3).

(3) Let 'Julius' name the inventor of the zip.

Let $\mathrm{ZIP}=\ulcorner$ there is a unique inventor of the ZIP $\urcorner$. For any context $c$ such that $\llbracket \mathrm{ZIP} \rrbracket^{c, w_{c}} \neq 1$ (any context where there is no unique inventor of the zip), both $\llbracket(4) \rrbracket^{c}$ and $\llbracket(5) \rrbracket^{c}$ are undefined.

(4) Julius is the inventor of the zip.

(5) Julius is dead in 1909.

Why? For any $\neg$ ZIP context $c$, the stipulation fails to fix a meaning or content for 'Julius'-i.e., $\llbracket J u l i u s \rrbracket^{c}$ is undefined. That is to say:

$$
\llbracket J u l i u s \rrbracket=\lambda c: \llbracket \mathrm{ZIP} \rrbracket^{c, w_{c}}=1 . \lambda w . l x\left(x \text { invented the zip in } w_{c}\right)
$$

While it is plausible that implicitly defined terms have characters (or something close enough), these characters are partial - they have intensions only at contexts where the definition's presupposition is met. Sentences (4) and (5) have worldevaluable content only at ZIP contexts. So sentences (4) and (5) meet condition (SP2).

\section{An argument for (AK)}

We argue for (AK) on the following grounds. If $\phi$ semantically presupposes $\psi$, then, by Definition 1, either (SP1) or (SP2) holds for $\phi$ and $\psi$. In either case, given an independently plausible closure assumption, $\mathcal{K}_{\mathcal{A}} \phi$ implies $\mathcal{K}_{\mathcal{A}} \psi$.

\section{1 (AK) from (SP1)}

Suppose $\phi$ semantically presupposes $\psi$, via (SP1). For convenience, we introduce an ordinary necessity modal $(\square)$, with this interpretation: ${ }^{6}$

Definition $2 \quad \llbracket \square \chi \rrbracket^{c, w}=1$ iff $\forall v\left(\llbracket \chi \rrbracket^{c, v}=1\right)$ Notice that (6) and (7) together entail the relevant instance of (AK).

(6) If $\mathcal{K}_{\mathcal{A}} \square(\phi \rightarrow \psi)$, then $\mathcal{K}_{\mathcal{A}} \phi \rightarrow \mathcal{K}_{\mathcal{A}} \psi$

(7) $\mathcal{K}_{\mathcal{A}} \square(\phi \rightarrow \psi)$

I will now argue that (6) and (7) hold of $\phi$ and $\psi$.

Argument for (6). (6) is an independently plausible closure claim. For, suppose that $\mathcal{K}_{\mathcal{A}} \square(\phi \rightarrow \psi)$ and that $\mathcal{K}_{\mathcal{A}}(\phi)$. Notice the plausibility of (8).

(8) $\mathcal{K}_{\mathcal{A}}(\{\phi, \square(\phi \rightarrow \psi)\} \models \psi)$

\footnotetext{
5 I think it is fairly clear they satisfy standard linguistic tests for presupposition (see, e.g., the data in Sect. 4.4). So I will here argue only that (SP2) holds for them.

6 This definition is revised slightly in Sect. 4.3.3.
} 
Given something like the kind of epistemic closure defended in Williamson (2002), it seems one could have a knowledge-sufficient reason for $\psi$, consisting of one's knowledge-sufficient, a priori reasons for believing that $\square(\phi \rightarrow \psi)$, that $\phi$, and that $\{\phi, \square(\phi \rightarrow \psi)\} \models \psi$. In other words, one would have knowledge-sufficient, a priori reasons for believing each premise of the following argument.

1. $\phi$

2. $\square(\phi \rightarrow \psi)$

3. $\{\phi, \square(\phi \rightarrow \psi)\} \models \psi$

4. Therefore: $\psi$

Suppose $S$ knows the premises of this argument a priori, "makes" the argument, and so comes to believe $\psi$. This certainly seems to suffice for $S$ to know that $\psi$. What kind of knowledge does $S$ 's belief that $\psi$ comprise? Well, what is the nature of $S$ 's grounds for believing that $\psi$ ? A posteriori? Evidently not: $S$ makes an inference (knowable a priori to be valid) from premises themselves known to be a priori. Insofar as epistemic warrant does transmit, there is no reason to think that, in being transmitted, it should change character from a priori to a posteriori in the process. (I am supposing, as is fairly standard in discussions of inferential warrant, that in such a case $S$ 's warrant for believing $\psi$ does not involve a warrant for believing that she has, as a matter of psychological fact, performed the relevant inference.) In such a case, then, $S$ would have a knowledge-sufficient, a priori reason for believing $\psi-S$ would know a priori that $\psi$. Since $S$ is a possible agent, follows that $\mathcal{K}_{\mathcal{A}} \psi$.

Argument for (7). (7) is also plausible. Certainly, it can be known that $\square(\phi \rightarrow \psi)$. Notice:

$$
\llbracket \square(\phi \rightarrow \psi) \rrbracket^{c, w}=1 \quad \text { iff } \quad \forall v\left(\llbracket \psi \rrbracket^{c, v}=1, \quad \text { if } \llbracket \phi \rrbracket^{c, v}=1\right)
$$

Since, by supposition, $\phi$ and $\psi$ satisfy (SP1), it follows that for all $v$, if $\llbracket \phi \rrbracket^{c, v}$ has any value at all, then $\llbracket \psi \rrbracket^{c, v}=1$. So, a fortiori, we know that $\square(\phi \rightarrow \psi)$.

What needs to be shown is that this knowledge is a priori. Here there is no knockdown argument. But consider an example. ${ }^{7}$ Suppose that $\phi$ and $\psi$ are the following sentences.

$(\phi)$ The king of France is bald.

$(\psi)$ There is a unique king of France.

$\phi$ and $\psi$ satisfy (SP1), and it is quite plausible in this case that $\mathcal{K}_{\mathcal{A}} \square(\phi \rightarrow \psi)$. Although this is not an argument that (7) is in general true (i.e., true for any $\phi$ and

\footnotetext{
7 Examples like this are, of course, not the most solid basis for establishing a general claim like (7). If the reader does not find the arguments given for the plausibility of (7) persuasive, I invite him or her to restrict the scope of the claim to cases where it seems to him or her to hold. Such a reader, if s/he is inclined to accept the possibility of a priori knowledge in any case, will, I expect, agree that the applications in this paper in which some sentence $\phi$ is seen to (SP1)-presuppose $\psi$ are such that $\mathcal{K}_{\mathcal{A}} \square(\phi \rightarrow \psi)$. This sort of reader will then be able to appreciate the uses to which (AK) is put here, even while suspending judgment about the extent of further applications of the principle in cases involving different kinds of (SP1)-presupposition. Similar remarks apply to my arguments for (11) below.
} 
$\psi$ ), it will be good enough for our purposes: all (SP1)-related uses of (AK) in this paper can be justified by appeal to this sort of intuitive argument.

Nevertheless: I know of no cases of formulae satisfying (SP1) for which this general pattern of argument does not hold. This is not entirely unexpected. It does not seem unreasonable to require as a condition of linguistic competence that an agent know which conditions need to hold in a situation if her utterance is to be evaluable for truth with respect to that situation. Items of knowledge required for linguistic competence are generally taken to be items of a priori knowledge. This casts (7) in a plausible light.

\section{$3.2(\mathrm{AK})$ from (SP2)}

Now suppose $\phi$ semantically presupposes $\psi$, via (SP2). Let must be an operator of epistemic necessity, with this rudimentary, contextualist interpretation:

Definition $3 \llbracket$ must $\chi \rrbracket^{c, w}=1$ iff for all $v$ such that, for all that is known at $\langle c, w\rangle, v$ might be the actual world, $\llbracket \chi \rrbracket^{c, v}=1$. Notice that (10) and (11) together entail the relevant instance of $\mathrm{AK}^{8}$

(10) If $\mathcal{K}_{\mathcal{A}}$ must $(\phi \rightarrow \psi)$, then $\mathcal{K}_{\mathcal{A}} \phi \rightarrow \mathcal{K}_{\mathcal{A}} \psi$

(11) $\mathcal{K}_{\mathcal{A}}$ must $(\phi \rightarrow \psi)$

Argument for (10). According to (10), if it's knowable a priori that must $(\phi \rightarrow \psi)$ and that $\phi$, it is knowable a priori that $\psi$. Noticing the plausibility of (12)...

$$
\mathcal{K}_{\mathcal{A}}(\{\phi, \text { must }(\phi \rightarrow \psi)\} \models \psi)
$$

...we can establish (10) by running roughly the same argument that was run for the closure claim (6).

Argument for (11). What about (11)? Here, again, there is no knockdown argument, though an example is suggestive. Suppose $\phi$ and $\psi$ are the following sentences.

$(\phi)$ Julius is the inventor of the zip. [or, Julius is dead in 1909.]

$(\psi)$ There is a unique inventor of the zip.

As argued in Sect. 2, $\phi$ and $\psi$ satisfy (SP2). Moreover, for this case, it is quite plausible that $\mathcal{K}_{\mathcal{A}}$ must $(\phi \rightarrow \psi)$. Suppose we are in a context $c$ where 'Julius' receives the stipulative definition in question. We reason as follows:

To falsify must $(\phi \rightarrow \psi)$, there must be an epistemically possible world where $(\phi \wedge \neg \psi)$. But, for $\phi$ to even express an intension at $c, \psi$ must be true at the

\footnotetext{
${ }^{8}$ Compared to the rather direct argument that (AK) follows from (SP1), this is relatively convoluted. But the complications are necessary: although (5) (SP2) presupposes ZIP, it's not knowable a priori that $\square$ ((5) $\rightarrow$ ZIP) (because it is false that $\square((5) \rightarrow$ ZIP), since there is a possible world where Julius, the actual inventor of the zip, is dead in 1909 and zips do not exist). It is, however, as I argue below, plausibly knowable a priori that, if Julius died in 1909, there must have been a unique inventor of the zip.

9 Although some deny that $\{\phi$, must $(\phi \rightarrow \psi)\} \models \psi$, there are good (ostensibly a priori) arguments in favor of it. For some of these, see von Fintel and Gillies (2012).
} 
"actual" world (namely, the world in which the context is located). Ipso facto, any $(\phi \wedge \neg \psi)$ world is not, given what we know, a candidate for actuality. So $\operatorname{must}(\phi \rightarrow \psi)$.

The argument is valid, and each premise of the argument seems to be known a priori. The conclusion, then, seems to be knowable a priori.

Although this is not an argument that (11) is in general true, it will be enough for our purposes: all (SP2)-related uses of (AK) in this paper are justifiable by appeal to this sort of intuitive argument.

Less modestly, however, it seems reasonable to require as a condition of linguistic competence that an agent know which conditions actually need to hold in a context if her utterance is to express a proposition at that context. This casts (11) in a plausible light.

\section{Application}

\subsection{The necessary a posteriori}

Our first application uses (AK) to give a Kripkean-friendly resolution of a familiar puzzle about proper names. The puzzle is this. Assume a basic Kripkean semantics for names, so that 'Cicero' and 'Tully' are co-intensional (where $\alpha$ and $\beta$ are cointensional iff $\llbracket \alpha \rrbracket^{c}=\llbracket \beta \rrbracket^{c}$ ).

$\llbracket$ Cicero $\rrbracket^{c}=\lambda w$. Cicero

$\llbracket$ Tully $\rrbracket^{c}=\lambda w$. Tully $[=$ Cicero $]$

As is well-known, the Kripkean semantics conjoined with two widely accepted assumptions about a priori access to the meanings of our words entails an absurdity, namely, that it is knowable a priori that Cicero=Tully.

1. If $\alpha$ 's intension is $h$, it's knowable a priori that $\alpha$ 's intension is $h$ (Meaning Apriorism I) $\left[\forall c, \alpha, h\right.$ : if $\llbracket \alpha \rrbracket^{c}=h$, then $\left.\mathcal{K}_{\mathcal{A}} \llbracket \alpha \rrbracket^{c}=h\right]$

2. If $\alpha$ and $\beta$ are co-intensional, this fact is knowable a priori (Meaning Apriorism II) $\left[\forall c, \alpha, \beta\right.$ : if $\left.\llbracket \alpha \rrbracket^{c}=\llbracket \beta \rrbracket^{c}, \mathcal{K}_{\mathcal{A}}\left(\llbracket \alpha \rrbracket^{c}=\llbracket \beta \rrbracket^{c}\right)\right]$

3. So, $\mathcal{K}_{\mathcal{A}} \llbracket$ Cicero $\rrbracket^{c}=\lambda w$.Cicero, and $\mathcal{K}_{\mathcal{A}} \llbracket$ Tully $\rrbracket^{c}=\lambda w$.Tully. (1)

4. So, $\mathcal{K}_{\mathcal{A}} \llbracket$ Cicero $\rrbracket^{c, w}=$ Cicero, and $\mathcal{K}_{\mathcal{A}} \llbracket$ Tully $\rrbracket^{c, w}=$ Tully. (3)

5. Since $\lambda w$.Cicero $=\lambda w$. Tully, $\mathcal{K}_{\mathcal{A}} \llbracket$ Cicero $\rrbracket^{c}=\llbracket$ Tully $\rrbracket^{c}$. (2)

6. So, $\mathcal{K}_{\mathcal{A}}$ Cicero=Tully. $(4,5)$

Although metaphysically necessary, Cicero's identity with Tully is, of course, something knowable only a posteriori. So this argument is a reductio of one of its assumptions. The Kripkean, presumably pivoting off her commitment to her semantic theory, will tend to regard it as a reductio of at least one form of Meaning A Priorism. Various meaning apriorists (e.g., neo-Fregeans and twodimensionalists of various stripes) are inclined to respond to this sort of argument by rejecting the Kripkean semantics (in particular, its suggestion that the intension 
of a name conventionally encodes no descriptive, or otherwise conceptual, content).

This assessment of the dialectic is far too hasty. The Kripkean need not (indeed, should not) regard the argument as a reductio of Meaning Apriorism. She should, instead, regard the argument as a reductio of the "basic Kripkean semantics" for names proposed above. Other forms of the Kripkean semantics-ones that properly take into account the presuppositional content of proper names-are available. The argument shows only that a different such form of the Kripkean semantics is required.

Geurts (1997) (among others) has shown that an occurrence of a proper name $\epsilon$ bears a linguistic presupposition that $\epsilon$ designates a unique individual (formally, helping ourselves to some notation, that $\exists ! x[\Delta(\epsilon, x)]$, where ' $\Delta(\epsilon, e)$ ' expresses a designation relation between a name $\epsilon$ and an entity $e$ ). This presupposition is plausibly typed as an (SP1) semantic presupposition. Consider the sentence 'Cicero authored De Finibus'. What truth-value does this sentence have at worlds where 'Cicero' fails to designate a unique entity? The same truth-value, plausibly, that 'the king of France is bald' has at worlds where there is no unique king of France, i.e., no truth-value at all. Truth-value intuitions for non-referring descriptions and nonreferring names are on a par.

What this suggests is that the intension of a proper name $\epsilon$ is partial in roughly the way that the intension of a definite description is partial. We might, for instance, write a lexical entry for a name $\epsilon$ thus:

$$
\llbracket \epsilon \rrbracket^{c}=\lambda w: \llbracket\ulcorner\exists ! x[\Delta(\epsilon, x)]\urcorner \rrbracket^{c, w_{c}}=\llbracket\ulcorner\exists ! x[\Delta(\epsilon, x)]\urcorner \rrbracket^{c, w}=1 . l y\left[\Delta(\epsilon, y) \text { in }\left\langle c, w_{c}\right\rangle\right]
$$

On this proposal, the intension of $\epsilon$ would be a partial function mapping all and only those worlds $w$ such that $\epsilon$ uniquely designates (both at the actual context and at $w$ ) to the entity that $\epsilon$ uniquely designates at the actual context. ${ }^{10}$

This semantics can easily be made consonant with the spirit of a Kripkean semantics for proper names. If $e$ is the individual uniquely designated by $\epsilon$ at the actual world, we can write a Krikpean lexical entry for $\epsilon$ thus:

$$
\llbracket \epsilon \rrbracket^{c}=\lambda w: \llbracket\left\ulcorner\exists ! x[\Delta(\epsilon, x)] \rrbracket^{c, w_{c}}=\llbracket\ulcorner\exists ! x[\Delta(\epsilon, x)]\urcorner \rrbracket^{c, w}=1 . e\right.
$$

Is such a semantics rightfully called Kripkean? Yes.

- Though $\epsilon$ will not super-rigidly designate its referent (i.e., at every world simpliciter), it will rigidly designate it (at all and only those worlds where something can lay unique claim to being designated by $\epsilon$ ). That is hardly surprising. Compare the commonplace view (defended by Kripke himself in Naming and Necessity) that rigid designation by $\epsilon$ of $e$ requires only that $\epsilon$ designate $e$ at all (and only) those worlds where e exists.

\footnotetext{
10 Swanson (2006) argues, contra Geurts (1997), that the fact that proper names bear descriptive presuppositions is compatible with a Kripkean semantics for proper names. For an argument in a similar spirit, see directly below. I note in passing that we might also wish to encode the presupposition at the hyperintensional level, so that $\llbracket \epsilon \rrbracket$ would be defined at $c$ only when $\epsilon$ designated a unique individual at $\left\langle c, w_{c}\right\rangle$. That seems plausible to me, but I will pass over this issue here.
} 
- The "descriptive" presupposition of a name does not ultimately figure in semantically determining the name's referent at a world. Although names have descriptive presuppositional content which is represented lexically, their referents are given directly.

An example will help clarify the latter point. First let $\kappa=\ulcorner\exists ! x[\Delta($ Cicero, $x)\urcorner$ and $\tau=\ulcorner\exists ! x[\Delta($ Tully, $x)]\urcorner$. Letting $c$ be any context in which Cicero and Tully are used in the way we intend to use them (to refer, respectively, to Cicero and Tully), the following is, I contend, a plausible, and fully Kripkean, proposal for the intensions of 'Cicero' and 'Tully':

$$
\begin{gathered}
\llbracket \text { Cicero } \rrbracket^{c}=\lambda w: \llbracket \kappa \rrbracket^{c, w_{c}}=\llbracket \kappa \rrbracket^{c, w}=1 \text {. Cicero } \\
\llbracket \text { Tully } \rrbracket^{c}=\lambda w: \llbracket \tau \rrbracket^{c, w_{c}}=\llbracket \tau \rrbracket^{c, w}=1 \text {. Tully }
\end{gathered}
$$

The extension of Cicero at a world, when it has one, is rendered simply as 'Cicero' (rather than - as might be the case on a reference-fixing descriptivist account - 'the individual actually designated by the name Cicero').

From this Kripkean semantic proposal, it follows that the sentence 'Cicero=Tully' will, by Definition 1, (SP1)-presuppose at least $\kappa$ and $\tau$. But, since of course it cannot be known a priori that a (use of a) name (in a given context) designates a unique entity (or, indeed, any entity at all), neither $\kappa$ nor $\tau$ is knowable a priori. Hence, by $(\mathrm{AK})$, it is not knowable a priori that Cicero=Tully.

Those committed to Meaning Apriorism may, of course, explain the failure of the argument for the claim that it is knowable a priori that Cicero=Tully by appeal to the supposed falsity of the Kripkean semantics. But, here, we have an explanation of the a posteriori status of 'Cicero is Tully' that is fully consistent with the Kripkean semantics. That explanation is, additionally, well-motivated, wholly independently of the Kripkean semantics. The Kripkean is thus able to resist the argument to the conclusion that it is knowable a priori that Cicero=Tully, for reasons totally independent of her commitment to her semantic theory.

Where, exactly, does the argument go wrong, on this sort of explanation? Here is a tentative idea. Notice that, since $\kappa$ and $\tau$ express different propositions, Cicero and Tully are not, in fact, co-intensional at all (since they have different definednessconditions). Indeed, it is contingent that Cicero and Tully should have been used to designate any individual (having, as they do, distinct associated causal chains). The intensions of Cicero and Tully therefore have distinct modal profiles: there are many circumstances relative to which $\llbracket$ Cicero $\rrbracket^{c}$ is defined, but $\llbracket$ Tully $\rrbracket^{c}$ is not.

To sum up, if our toy Kripkean proposal here is right, then:

- Intensions will, in some sense, conventionally encode descriptive (on our proposal, metalinguistic) content.

- However, this conventional encoding will occur at the presuppositional level, rather than at the level of (proffered) content or reference. Names proffer nothing more than their referents; the conditions under which they succeed or fail to proffer a referent are, however, descriptively rich. (Which is not, of course, to say that the mode of determining a name's referent is descriptively rich.) 
- Meaning Apriorism may well be false-indeed, I think (at least) Meaning Apriorism II most assuredly is false-but this argument itself puts no pressure on the Kripkean to say this. The Kripkean's "explanation" for why the argument is unsound is of roughly the same shape as that of, e.g., the neo-Fregean: Cicero and Tully are not co-intensional. The neo-Fregean, however, locates this failure of co-intensionality in the proffered contents of the names Cicero and Tully, whereas the Kripkean locates it in their non-proffered, presuppositional content.

In short, both theories successfully explain the a posteriori status of Cicero's identity to Tully. The a posteriori identity of Cicero and Tully is, therefore, orthogonal to the debate between the Kripkean and her opponent. ${ }^{11}$

\subsection{Analyticity}

It is natural to think it is knowable a priori that the queen of England is a queen of England, or that the queen of England is self-identical. But if the lexical proposals for the meaning of the definite determiner described in Sect. 2.1 are correct, this can't, strictly speaking, be correct. According to these proposals, (13) and (14) bear the semantic presupposition that (15) is true.

(13) The queen of England is a queen of England.

(14) The queen of England is the queen of England.

(15) There is a unique queen of England.

But (15) is not knowable a priori. So, by (AK), neither are (13) or (14). Interestingly, if $(\mathrm{AK})$ is right, the a priori knowability of certain claims depends on the fate of certain theses in natural language semantics (in this case, a popular semantics for the definite determiner). ${ }^{12}$

Although this might seem like a pedantic complaint, it is actually rather surprising, since the obvious natural logical forms for (13) and (14)-(16) and (17), respectively_are logical truths.

(16) $Q(\iota x Q(x))$

(17) $\iota x Q(x)=\iota x Q(x)$

This suffices to make (13) and (14) analytic, according to some characterizations of analyticity, e.g., Boghossian's (1996) "Frege-Analyticity". (AK) provides a new template for generating examples of the analytic a posteriori.

\footnotetext{
11 A residual puzzle for the Kripkean is to explain why it cannot be known a priori that $(\kappa \wedge \tau) \rightarrow$ Cicero=Tully. I deal with this in other work.

12 Russellianism about definite descriptions does not avoid these results, since Russellianism, if anything, makes it more plausible that $\mathcal{K}_{\mathcal{A}} \square[(13) \rightarrow(15)]$. According to the Russellian, (13)'s meaning is given by a conjunction, one of whose conjuncts is (15).
} 


\subsection{Filtering}

Semantic presuppositions can be syntactically filtered or cancelled (a.k.a. locally satisfied) under certain kinds of embedding. In view of (AK), it will become clear that presupposition-filtering has significant epistemological implications.

\subsubsection{Conditionals}

One way to filter a sentence's presuppositions is to embed it as the consequent of a conditional whose antecedent entails its presuppositions (Heim 1991). Formally, if $\phi$ presupposes that $\psi_{1}, \ldots, \psi_{n}$ and, for all $i, 1 \leq i \leq n, \chi$ entails $\psi_{i}$, there will be a salient reading of a conditional $\ulcorner\chi \rightarrow \phi\urcorner$ that does not presuppose $\psi_{i}$, for any $i$, $1 \leq i \leq n .^{13}$ (18) gives a simple example.

(18) If there is a unique king of France, the king of France is male.

Such readings of such conditionals are known as LOCAL SATISFACTION readings. ${ }^{14}$

Thus, while (13) is not knowable a priori, if we filter the presupposition of (13) that $(15)$ is true, by forming a conditional of the form $\ulcorner(15) \rightarrow(13)\urcorner$, we achieve something that (AK) does not claim to be knowable only a posteriori.

Interestingly, philosophical work on the a priori betrays a fairly systematic tendency to filter a posteriori presuppositions in exactly this way. To my knowledge, none of this work construes this tactic as presupposition-filtering. (AK) both explains and legitimates this systematic tendency, in a very tidy way.

Evans (1985) and Hawthorne (2002), for example, do not cite (4) as an example of the contingent a priori, but rather (19).

(19) If anyone uniquely invented the zip, Julius invented the zip.

This is a standard reaction to Kripke's original argument for the contingent a priori. Evans, for his part, writes that (4) "requires for its truth something which ... [(19)] does not, namely that someone did uniquely invent the zip, and since this cannot be known a priori, neither can [(4)]" (Evans 1985, p. 193). There is, of course, a gap in Evans' argument here, which (AK) fills in nicely.

\subsubsection{Projection}

PROJECTION is the flipside of local satisfaction; presuppositions project out of embedded constructions if they are not locally satisfied. We thus predict (correctly, I suggest) that $\llbracket(20) \rrbracket^{c}$ is undefined over any $w$ where there is not a unique king of France, and that $\llbracket(21) \rrbracket$ is undefined over any $c$ such that no one uniquely invented the zip in $c$ 's world.

\footnotetext{
${ }^{13}$ It is sometimes suggested that conditionals presuppose that their antecedents are possible with respect to the conversational context. Since this will not count as a semantic presupposition, we can safely ignore it.

${ }^{14}$ See Heim (1991). It's generally agreed that any presupposition of a conditional's antecedent must be borne by the entire conditional (i.e., it projects to the entire conditional).
} 
(20) \#If the king of France is bald, he doesn't own a comb.

(21) \#If Julius died in 1909, he knew of McKinley's assassination. (as interpreted at a context in a world lacking a unique inventor of the zip)

Suppose $\phi$ presupposes $\chi$, and consider a conditional embedding $\phi$ as its antecedent. Since nothing in this conditional locally satisfies the presupposition of its antecedent, the conditional must be embedded under something else for $\phi$ 's presuppositions to be locally satisfied. If the conditional is unembedded, its semantic presuppositions must be globally satisfied (i.e., by the context or world of evaluation) for the conditional to be true. ${ }^{15}$ There are two notable epistemological upshots here.

(E1) If $\ulcorner\phi \rightarrow \psi\urcorner$ is unembedded and $\psi$ semantically presupposes $\chi$, then if $\chi$ is not locally satisfied (entailed by $\phi), \mathcal{K}_{\mathcal{A}}(\phi \rightarrow \psi)$ only if $\mathcal{K}_{\mathcal{A}} \chi$.

(E2) If $\ulcorner\phi \rightarrow \psi\urcorner$ is unembedded and $\phi$ semantically presupposes $\chi$, then $\mathcal{K}_{\mathcal{A}}(\phi \rightarrow$ $\psi)$ only if $\mathcal{K}_{\mathcal{A}} \chi$.

\subsubsection{Modals}

(E2) might seem to make trouble for our argument for (AK). In Sects. 3.1 and 3.2, I claimed that (22) and (23) were knowable a priori.

(22) $\square$ (if the king of France is bald, there is a unique king of France)

(23) must (if Julius is dead in 1909, someone uniquely invented the zip)

\footnotetext{
15 For simplicity, this formulation (and much of my subsequent discussion) deliberately ignores cases where presuppositions are satisfied, in whole or in part, by induced contexts, rather than by syntactic material.
}

A: France might have exactly one king.

B: Well, then the King of France is male.

[Modal Subordination; cf. Roberts (1989)]

France has at least one king. If it has no more than one, the King of France is male.

[Partial Local, Partial Global Satisfaction]

In neither case does the speaker of the sentence in which the definite noun phrase 'the King of France' appears presuppose that France has a unique king. Nevertheless, in neither case is the presupposition either globally or locally satisfied. These are well-attested facts that I am happy to grant.

Does it affect my position on the epistemological status of conditionals? It does not. The proposition normally expressed by the speaker's sentence ('the King of France is male' or 'If France has no more than one king, the King of France is male') is not knowable a priori (nor, I think, would we even wish to ascribe such knowledge, a priori or a posteriori, to the speaker).

It is true that, in such cases, an individual does seem to be hitting on a specific item of a priori knowledge, namely:

If France has exactly one king, the King of France is male.

But my view on the epistemological status of conditionals survives intact: the thing that the speaker can know a priori cannot itself have any semantic presuppositions that are not knowable a priori. The speaker cannot know a priori that the King of France is male, or that: if France has no more than one king, the King of France is male. What the speaker can know a priori is a conditional where the antecedent locally satisfies the presupposition expressed by the consequent. (It follows that such cases do not represent counterexamples to (E1) and (E2) below.) Thanks to an anonymous reviewer for pressing me on these points. 
The antecedents of the embedded conditionals carry semantic presuppositions whose truth cannot be known a priori. So, by (E2), it would seem, contrary to my claims, that (22) and (23) are not knowable a priori.

Crucially, however, these conditionals are embedded under modal operators. Quantificational adverbs (and their logical counterparts) can (within limits) neutralize presuppositions of sentences over which they take scope (cf. Geurts 1997). Although the precise manner in which this happens is not important for our purposes here, it may help the reader to consider an example:

(24) Necessarily, the number of the planets is odd

Such a sentence has two salient readings: de dicto and de re. On the de re reading, the sentence presupposes that there are some planets which can be counted up to some natural number $n$ (let us be generous with Pluto and say that $n=9$ ), and asserts (truly) of $n$ that $n$ is necessarily odd. On the de dicto reading, the presupposition of the definite noun phrase 'the number of planets' is neutralized (specifically, it restricts the domain of quantification for the modal adverb to possibilities in which there are some planets which can be counted up to some natural number $k$ ), and the sentence asserts something false (that for every world $w$ in this domain, $k$ is odd at $w$ ).

In common philosophical parlance, we might say that the modal operator in, e.g., (22) makes available a de dicto reading of 'the king of France.' This is obvious enough, since (22) is perfectly assertable at contexts where it's common ground that France is not a monarchy, while (20) is not. Notice, then, that (AK)-driven objections to the a priori knowability of (13) and (14) can be sidestepped by affixing a necessity operator to the front of these sentences, as follows:

$\square$ (The queen of England is a queen)

(26) $\square$ (The queen of England is the queen of England)

On their de dicto readings, the resulting sentences bear no offending semantic presuppositions. Plausibly they are knowable a priori.

De dicto readings introduce a minor semantic complication. Given that there are worlds at which the embedded conditional in (22) is not true (because neither true nor false), the semantics for $\square$ stated in Definition 2 must be revised if we are to predict a true de dicto reading of (22). The shape of the required revision is fairly clear: in de dicto readings, the neutralized presuppositions function to restrict the domain over which $\square$ and must quantify: the de dicto reading of (22) is true iff all worlds in which there is a unique king France are such that: if the unique king of France is bald, there is a unique king of France. Similarly for (23). ${ }^{16}$

Surface form is typically ambiguous between de dicto and de re readings. That is to say: whether a presupposition-carrying expression embedded under a modal operator is to be interpreted de dicto (so that its presuppositions are interpreted as domain restrictors rather than felicity-conditions) or de re (so that its

\footnotetext{
16 This mirrors the standard semantics for sentences of forms $\ulcorner\square a=a\urcorner$ and $\ulcorner\square P(a)\urcorner$, where $a$ is a singular term and $P$ a predicate. These sentences are generally regarded as true iff $\ulcorner a=a\urcorner$ and $\ulcorner P(a)\urcorner$ are, respectively, true at every world where a refers (not at every world simpliciter).
} 
presuppositions project to the whole sentence) is not settled by the surface form of the sentence in which it occurs. There is, for instance, a false reading of (25), with roughly the logical form given in (27). Similarly, there is no true reading of (28) on which 'the king of France' is given a projection (de re) reading, as represented in (29), since, on such a reading, the semantic presupposition of the definite description is neither locally nor globally satisfied. ${ }^{17}$

(27) [the queen of England $]_{i}\left[\square\right.$ ( $t_{i}$ is a queen of England)]

(28) $\square$ (the king of France is male)

(29) [the king of France $]_{i}\left[\square\left(t_{i}\right.\right.$ is male $\left.)\right]$

These comments are all rather general. The subsequent section explores a more specific epistemological upshot of presupposition-filtering.

\subsection{The contingent a priori}

The following facts conspire to create trouble for the classic Kripkean argument for the contingent a priori.

(F1) If $\ulcorner\phi \rightarrow \psi\urcorner$ is unembedded and $\psi$ semantically presupposes that $\chi$, then if $\chi$ is not locally satisfied (entailed by $\phi), \mathcal{K}_{\mathcal{A}}(\phi \rightarrow \psi)$ only if $\mathcal{K}_{\mathcal{A}}$.

(F2) A proper name $\epsilon$ carries the semantic presupposition that $\exists ! x[\Delta(\epsilon, x)]$.

In Sect. 2.2, I argued that 'Julius' (and therefore 'Julius invented the zip') bears an (SP2) presupposition that $\mathrm{ZIP}_{1}$ is true. But, in view of (F2), it will also bear an (SP1) presupposition that $\mathrm{ZIP}_{2}$ is true.

( $\left.\mathrm{ZIP}_{1}\right) \exists ! x[$ invented the zip $(x)]$

( $\left.\mathrm{ZIP}_{2}\right) \exists ! x[\Delta($ Julius, $x)]$

By (F1), since neither $\mathrm{ZIP}_{1}$ nor $\mathrm{ZIP}_{2}$ is knowable a priori, the consensus "safe" item of putatively contingent a priori knowledge (19), repeated here as (30), is actually knowable a priori only if the presuppositions borne by its consequent ( $\mathrm{ZIP}_{1}$ and $\mathrm{ZIP}_{2}$ ) are both locally satisfied by its antecedent. (Recall the discussion of Sect. 4.3.1.)

(30) If anyone uniquely invented the zip, Julius invented the zip.

But, since the antecedent does not entail $\mathrm{ZIP}_{2}$, that presupposition will project, leading to the conclusion that (30) is not knowable a priori. The immediate fix is not, of course, a hard one: swapping (30) for (31) seems to do the trick.

(31) If anyone uniquely invented the zip and 'Julius' uniquely designates him, Julius invented the zip.

\footnotetext{
17 Per the usual syntactic convention, $t_{i}$ is a trace recording movement of the co-indexed noun phrase at logical form.
} 
I don't dispute the knowability a priori of (31); instead I wish to dispute its contingency. To be somewhat more precise, I will suggest that (31) is ambiguous between two kinds of readings. There is a reading on which the antecedent nullifies the a posteriori presupposition of the consequent (the de dicto reading), and there is a reading on which it does not (the de re reading). The former express a necessary truth, while the latter expresses a contingent truth. But the contingent reading is associated with the projection of the consequent's a posteriori presupposition: on this reading (31) presupposes $\left(\mathrm{ZIP}_{1}\right)$ and $\left(\mathrm{ZIP}_{2}\right)$. Applying $(\mathrm{AK})$, since these presuppositions are not knowable a priori, the contingent reading of (31) does not give something knowable a priori: (31) is, on this reading, contingent a posteriori. Since the other reading is necessary, there is no contingent a priori reading of (31).

The source of the trouble here is that genuine local satisfaction (de dicto) readings of definite noun phrases are typically non-referential in character. ${ }^{18}$ See, for instance, (32), (33), and the suggested (Discourse Representation Theoretic) glosses.

$$
\begin{aligned}
& \square \text { (the king of France is male) } \approx \\
& \square[[x \text { : unique king of } \operatorname{France}(x)] \Rightarrow[\operatorname{male}(x)]]
\end{aligned}
$$

(33) If there's a unique king of France, the king of France is male $\approx$ $[x$ : unique king of $\operatorname{France}(x)] \Rightarrow[\operatorname{male}(x)]$

Informally speaking, in evaluating (32), we conditionally or hypothetically introduce $^{19}$ a discourse referent $\boldsymbol{x}$ representing the unique king of France in a world $w$ and consider whether the individual the interpretation assigns to $\boldsymbol{x}$ at $w$ is male. We accept (32) as true if, for all $w$ such that an individual is assigned to $\boldsymbol{x}$ at $w$, it is male. Likewise, minus the quantification over worlds, for (33).

When interpretation of a definite noun phrase $\delta$ bearing a presupposition $\psi$ is tied to a conditionally introduced discourse referent containing the information that $\psi$, we will say (following the terminology of Geurts and his fellow travelers) that $\delta$ is given an anaphorically bound reading. Proper names admit of anaphorically bound readings, as (34) demonstrates. Exactly the same phenomenon can be seen to occur in (31), when 'Julius' is given an anaphorically bound reading, as in (35).

(34) If there's a tastiest pie and 'Ed' names it, then Ed is a pie $\approx$

$[\boldsymbol{y}$ : tastiest $\operatorname{pie}(y) \wedge$ named by 'Ed' $(y)] \Rightarrow[\operatorname{pie}(y)]$

(35) If anyone uniquely invented the zip and 'Julius' uniquely designates him, Julius invented the zip $\approx$

[z: uniquely invented the $\operatorname{zip}(z) \wedge$ uniquely designated by 'Julius'(z)] $\Rightarrow$ [invented the $\operatorname{zip}(z)$ ]

Clearly though the rendering in (35) does not yield a contingent reading of (31).

\footnotetext{
18 The explanation for this is intuitive enough — when we use a definite noun phrase referentially, we presuppose that there is something for it to designate or refer to.

19 Conditional introduction means that the discourse referent does not become part of the basic "structure" we use to represent or keep track of the conversational score. So long as 'the king of France' is read de dicto, utterances of (32) and (33) neither require nor make it the case that it is common ground that there is a unique king of France. This sort of device for generating anaphorically bound readings for such expressions is familiar from the Discourse Representation Theoretic treatment of Donkey Anaphora.
} 
Local satisfaction is the source of this difficulty. The contingent reading of (31) can only be obtained by giving 'Julius' a projection ( $d e$ re) reading. Let $C$ be a disambiguated sentence giving the contingent reading of (31). Since $C$ represents a de re reading of 'Julius', $C$ will semantically presuppose both $\mathrm{ZIP}_{1}$ and $\mathrm{ZIP}_{2}$. So, by (F1), $C$ is knowable a priori only if both $\mathrm{ZIP}_{1}$ and $\mathrm{ZIP}_{2}$ are. But they are not. So $C$ is not.

More generally, any sentence representing a contingent reading of (31) will semantically presuppose both $\mathrm{ZIP}_{1}$ and $\mathrm{ZIP}_{2}$. So, there is no contingent a priori reading of (31).

\subsection{Knowledge of logic}

At first blush, stipulative definition seems to generate easy a priori knowledge-a fact that has been enthusiastically exploited by philosophers since Naming and Necessity. But such knowledge is often less easy than it seems, as we've seen. I close this essay with another variation on this theme. Utilizing the fact that stipulative definitions apparently involve (SP2) presuppositions, I'll argue that (AK) is able to make trouble certain arguments for a priori knowledge of logic. Here, as before, the trouble has not gone unnoticed by others, ${ }^{20}$ though the ultimate source of the trouble-the phenomenon of semantic presupposition, and the epistemic commitments generated thereby-has.

Various philosophers are inclined to attempt to ground a priori knowledge of logic, by means of the following argument. ${ }^{21}$ Let $\mathbf{A}=\left\{\left(\wedge E_{1}\right),\left(\wedge E_{2}\right),(\wedge I)\right\}$, i.e., the set of introduction and elimination rules, in conditional form, for $\wedge$.

$$
\begin{aligned}
& \left(\wedge E_{1}\right)(p \wedge q) \rightarrow p \\
& \left(\wedge E_{2}\right)(p \wedge q) \rightarrow q \\
& (\wedge I) p \rightarrow(q \rightarrow(p \wedge q))
\end{aligned}
$$

The usual argument goes something like this. We first stipulate that $\wedge$ shall have whatever connective-appropriate meaning makes each conditional in A logically true (i.e., true under any propositional interpretation of $p$ and $q$ ). Having settled on this meaning for $\wedge$ by fiat, we automatically place ourselves in a position to know (a priori) that, e.g., an arbitrary instance of $\left(\wedge E_{1}\right)$ is true.

It's clear, however, that $\left(\wedge E_{1}\right),\left(\wedge E_{2}\right)$, and $(\wedge I)$ (and all instances thereof) each bear the (SP2) presupposition that $\sigma$ is true.

$$
(\sigma) \exists \mathfrak{F}: \wedge \text { means } \mathfrak{F} \text { and } \mathfrak{F} \text { makes }\left[\begin{array}{l}
\ulcorner(p \wedge q) \rightarrow p\urcorner \\
\ulcorner(p \wedge q) \rightarrow q\urcorner \\
\ulcorner p \rightarrow(q \rightarrow(p \wedge q))\urcorner]
\end{array}\right] \text { logically true. }
$$

For any $\neg \sigma$ context $c$, if $\wedge$ 's definition were (per impossibile) to occur in $c$, any instance of $\left(\wedge E_{1}\right)$ would fail to express a proposition in $c$. So, any instance of $\left(\wedge E_{1}\right)$

\footnotetext{
${ }^{20}$ See, e.g., Horwich (2000) for a line of attack similar in spirit to the one developed here.

21 Boghossian (1996) and Hale and Wright (2000) are some prominent proponents of roughly this kind of argument.
} 
bears an (SP2) presupposition that $\sigma$ is true. Therefore, if $\mathcal{K}_{\mathcal{A}}\left(\wedge E_{1}\right)$, then $\mathcal{K}_{\mathcal{A}} \sigma$. If it's false that $\mathcal{K}_{\mathcal{A}} \sigma$, it's false that $\mathcal{K}_{\mathcal{A}}\left(\wedge E_{1}\right)$. What goes for $\left(\wedge E_{1}\right)$ also goes for the other members of $\mathbf{A}$.

Since we are, inter alia, interested in grounding a priori knowledge of $\left(\wedge E_{1}\right)$, we will want to take extra care to rule it out that $\sigma$ is not knowable a priori. As it stands, we could do this two ways. First, using (AK), we could infer that $\mathcal{K}_{\mathcal{A}} \sigma$ from, e.g., $\mathcal{K}_{\mathcal{A}}\left(\wedge E_{1}\right)$. But, of course, it's illicit to assume that $\mathcal{K}_{\mathcal{A}}\left(\wedge E_{1}\right)$ for this purpose, since this is precisely what we're attempting to establish.

Alternatively, we could attempt an independent argument for $\mathcal{K}_{\mathcal{A}} \sigma$. But any argument along these lines would also be, a fortiori, an argument that $\mathcal{K}_{\mathcal{A}}\left(\wedge E_{1}\right)$. Far from alleviating the problem of grounding a priori knowledge of the logical truths in A, it seems we've only managed to restate it.

To their credit, Hale and Wright (2000) do seem to recognize a problem in this general vicinity. They argue (convincingly) that a stipulation that $\phi$ cannot per se yield any sort of knowledge unless $\phi$ can be known "without collateral... epistemic work" (p. 297). One cannot, for example, know (36) solely via stipulating (36), since knowledge of (36) requires that one do collateral epistemic work to verify that there is a (unique) such murderer. No such collateral epistemic work is required for knowledge of (37), they claim.

(36) Jack the Ripper is the murderer of Mary Ann Nichols.

(37) If there's a unique murderer of Mary Ann Nichols, Jack the Ripper is the murderer of Mary Ann Nichols.

Notice that, in the case of (36), the requisite "collateral" item of knowledge is the (SP1) presupposition of the definite description 'the murderer of Mary Ann Nichols'. With (AK) in hand, we have a clear explanation of why (36) requires such collateral epistemic work, and of why (37) does better: the relevant presupposition is locally satisfied. ${ }^{22}$

But Hale and Wright draw the wrong lesson from this case: that embedding a bare sentence $F(a)$ containing a stipulatively defined term $a$ in an unasserted environment (e.g., $\ulcorner F(a) \rightarrow \psi\urcorner$ or $\ulcorner\psi \rightarrow F(a)\urcorner$ ) suffices to neutralize any demand for collateral knowledge that the world cooperates in putting forth a suitable meaning for $a$. So, they claim, one need not be able to know $\sigma$ (a priori) in order to know $\left(\wedge E_{1}\right),\left(\wedge E_{2}\right)$, or $(\wedge I)$ (a priori), since, in all of these cases, $\wedge$ occurs in the antecedent or consequent of a conditional.

That is mistaken. As we have argued at length, presuppositions project out of unasserted environments unless they are locally satisfied. Because $\wedge$ bears the (SP2) presupposition that $\sigma$ is true, and because nothing in $\left(\wedge E_{1}\right),\left(\wedge E_{2}\right)$, or $(\wedge I)$ is up to locally satisfying this presupposition, all of these conditionals semantically presuppose $\sigma$, and so none are knowable a priori unless $\sigma$ is knowable a priori as well. This, for reasons already sketched, means that the proponent of this way of securing a priori logical knowledge incurs a commitment to make it independently plausible that $\sigma$ is knowable a priori. But this means having made no headway in the project of grounding a priori knowledge of logic at all.

\footnotetext{
22 I'm ignoring the semantic presupposition of 'Jack the Ripper' for simplicity's sake.
} 


\section{Conclusion}

Research into the possibility of certain sorts of a priori knowledge would benefit from close attention to the linguistic phenomenon of semantic presupposition. We've seen that (AK) has a rather wide range of application. This leads one to wonder whether (AK) might be usefully applied to other longstanding questions about the a priori. It would be surprising if it could not.

Acknowledgments For discussion of the ideas in this paper, I am grateful to Anders J. Schoubye, Eduardo García-Ramírez, Ofra Magidor, Eric Swanson, Ian Proops, to audiences at Oxford and Arché, and to an anonymous referee for this journal.

\section{References}

Boghossian, P. (1996). Analyticity reconsidered. Nô̂s, 30, 360-391.

Evans, G. (1985). Reference and contingency. In J. McDowell (Ed.), Collected papers (pp. 178-213). New York: Oxford University Press.

Geurts, B. (1997). Good news about the description theory of names. Journal of Semantics, 14, 319-348.

Hale, B., \& Wright, C. (2000). Implicit definition and the a priori. In P. Boghossian \& C. Peacocke (Eds.), New essays on the a priori (pp. 286-319). New York: Oxford University Press.

Hawthorne, J. (2002). Deeply contingent a priori knowledge. Philosophy and Phenomenological Research, 65, 247-269.

Heim, I. (1991). On the projection problem for presuppositions. In S. Davis (Ed.), Pragmatics (pp. 397-405). New York: Oxford University Press.

Heim, I., \& Kratzer, A. (1998). Semantics in generative grammar. Oxford: Blackwell.

Horwich, P. (2000). Stipulation, meaning, and apriority. In P. Boghossian \& C. Peacocke (Eds.), New essays on the a priori (pp. 150-170). New York: Oxford University Press.

Roberts, C. (1989). Modal subordination and pronominal anaphora in discourse. Linguistics and Philosophy 12, 683-721 doi:10.1007/BF00632602.

Swanson, E. (2006). Interactions with context. Ph.D. Dissertation, MIT, Cambridge, MA.

von Fintel, K. (2004). Would you believe it? The King of France is back! Presuppositions and truth-value intuitions. In M. Reimer \& A. Bezuidenhout (Eds.) Descriptions and beyond (pp. 269-296). New York: Oxford University Press.

von Fintel, K., \& Gillies, A. S. (2012). Must... stay... strong! Natural Language Semantics. doi: 10.1007/s11050-010-9058-2.

von Fintel, K., \& Heim, I. (2007). Intensional Semantics. Unpublished manuscript, MIT, Cambridge, MA. Williamson, T. (2002). Knowledge and its limits. New York: Oxford University Press. 